Int. J. Morphol.,

31(2):500-504, 2013.

\title{
Persistencia del Collar Venoso Renal
}

\author{
Persistence of Renal Venous Collar
}

Sousa- Rodrigues, C. F."; Alcântara, F. S."; Rocha, A. C.*; Gusmão, L. C."; Monte-Bispo, R. F."; Ferreira, A. K." \& Olave, E.*

SOUSA- RODRIGUES, C. F.; ALCÂNTARA, F. S.; ROCHA, A. C.; GUSMÃO, L. C.; MONTE-BISPO, R. F.; FERREIRA, A. K. \& OLAVE, E. Persistencia del collar venoso renal. Int. J. Morphol., 31(2):500-504, 2013.

RESUMEN: El desarrollo del sistema venoso cava es bastante complejo, pudiendo producirse innumerables variaciones de los padrones anatómicos ya conocidos, siendo algunos más frecuentes y otros de rara incidencia. De estas variaciones, puede ocurrir una en que se forma un anillo vascular en torno de la aorta, constituído por una vena renal pre-aórtica y otra retro-aórtica, desembocando a un nivel más bajo en la vena cava inferior (VCI), una disposición llamada "collar venoso renal", que consiste en la persistencia de las anastomosis intersupracardinales e intersubcardinales embrionarias. En la variación presentada en este artículo, la disposición de los vasos corresponde a un tipo de la clasificación mencionada en la literatura, con excepción de la emergencia independiente de los componentes pre y retroaórtico del collar referido, a partir del hilio renal y del calibre distal de la vena renal retroaórtica. La vena renal preaórtica tenía $90 \mathrm{~mm}$ de longitud y $20 \mathrm{~mm}$ de calibre en su parte terminal, desembocando en la VCI a nivel del tercio inferior de la vértebra L1. La vena renal retroaórtica tenía $125 \mathrm{~mm}$ de longitud, cruzando las vértebras L1 y L2 para desembocar en la VCI a nivel del tercio superior de L3, donde se registró un diámetro terminal de $14 \mathrm{~mm}$. La disposición presentada, es una variación potencialmente peligrosa de la vena renal izquierda, importante de recalcar su presencia, ya que ha sido relatado que en cirugías retroperitoneales no se ha identificado el componente dorsal, produciendo hemorragia profusa, nefrectomía innecesaria y hasta la muerte.

PALABRAS CLAVE: Riñón; Venas renales; Variación anatómica.

\section{INTRODUCTION}

El desarrollo del sistema venoso cava es muy complejo, dando lugar a numerosas variaciones en los padrones anatómicos. Las variaciones de las venas renales no son comunes, pudiendo ocurrir raramente en el lado izquierdo $\mathrm{y}$, aún dentro de este grupo, formar un anillo vascular en torno de la aorta, donde se puede observar una vena renal pre-aórtica y otra, retro-aórtica, disposición descrita como persistencia del collar renal (Williams et al., 1995).

Según Gardner et al. (1988), las venas renales anómalas no son comunes. Las venas renales múltiples se presentan ocasionalmente, encontrándose comúnmente en el lado derecho (Hollinshead, 1962). La vena renal izquierda es raramente múltiple, sin embargo puede dividirse y formar el anillo circunaórtico (Gardner et al.; Hollinshead), señalando el último autor que en el lado derecho, la multiplicidad de las venas renales es tan frecuente como las arterias, siendo más rara en el lado izquierdo.

En el presente trabajo se relata una disposición de innumerables y simultáneas variaciones vasculares, parti- cularmente de las venas renales, resultantes de una mal-formación embrionaria, analizándose las implicancias que cada una de éstas podría tener durante una eventual cirugía o en la propia fisiología de un individuo.

\section{MATERIAL Y MÉTODO}

Las variaciones vasculares fueron observadas en un cadáver de un individuo brasileño, adulto, de sexo masculino, fijado en formaldehido al $10 \%$, durante una disección realizada en el Laboratorio de Anatomía Topográfica del Centro de Ciencias Biológicas de la Universidad Federal de Alagoas, Brasil.

Para mejor visualización del campo en cuestión, se procedió a separar las asas intestinales, sección del peritoneo parietal posterior y la retirada de la grasa pararenal, pudiendo observar la variante de la disposición vascular.

\footnotetext{
* Departamento de Morfologia, Centro de Ciencias Biológicas, Universidade Federal de Alagoas, Maceió, Brasil.

*** Facultad de Medicina, Universidad de La Frontera, Chile.
} 
SOUSA- RODRIGUES, C. F.; ALCÂNTARA, F. S.; ROCHA, A. C.; GUSMÃo, L. C.; MONTE-BISPO, R. F.; FERREIRA, A. K. \& OLAVE, E. Persistencia del collar venoso renal. Int. J. Morphol., 31(2):500-504, 2013.

\section{RESULTADOS}

Las venas renales izquierdas pre y retro-aórtica se originaron separadamente del hilio renal, contribuyendo igualmente en la formación del pedículo renal, sin embargo, ellas se anastomosaron por medio de un corto tronco venoso de 2,0 $\mathrm{mm}$ de longitud y 4,0 $\mathrm{mm}$ de calibre, distante a $21 \mathrm{~mm}$ del hilio renal.

La vena renal pre-aórtica (Figs. 1 y 2) era más desarrollada que su correspondiente retro-aórtica (Fig. 2), teniendo $90 \mathrm{~mm}$ de longitud y un calibre de $20 \mathrm{~mm}$ en su terminación. Su desembocadura en la VCI fue típica, a nivel del tercio inferior de la vértebra L1.

La vena retro-aórtica midió $125 \mathrm{~mm}$ de longitud, siguiendo un trayecto descendente y oblicuo, cruzando las vértebras L1 y L2, para desembocar en la VCI por debajo de la vena renal pre-aórtica, a nivel del tercio superior de la vértebra L3, a $46 \mathrm{~mm}$ del inicio de la VCI. La misma

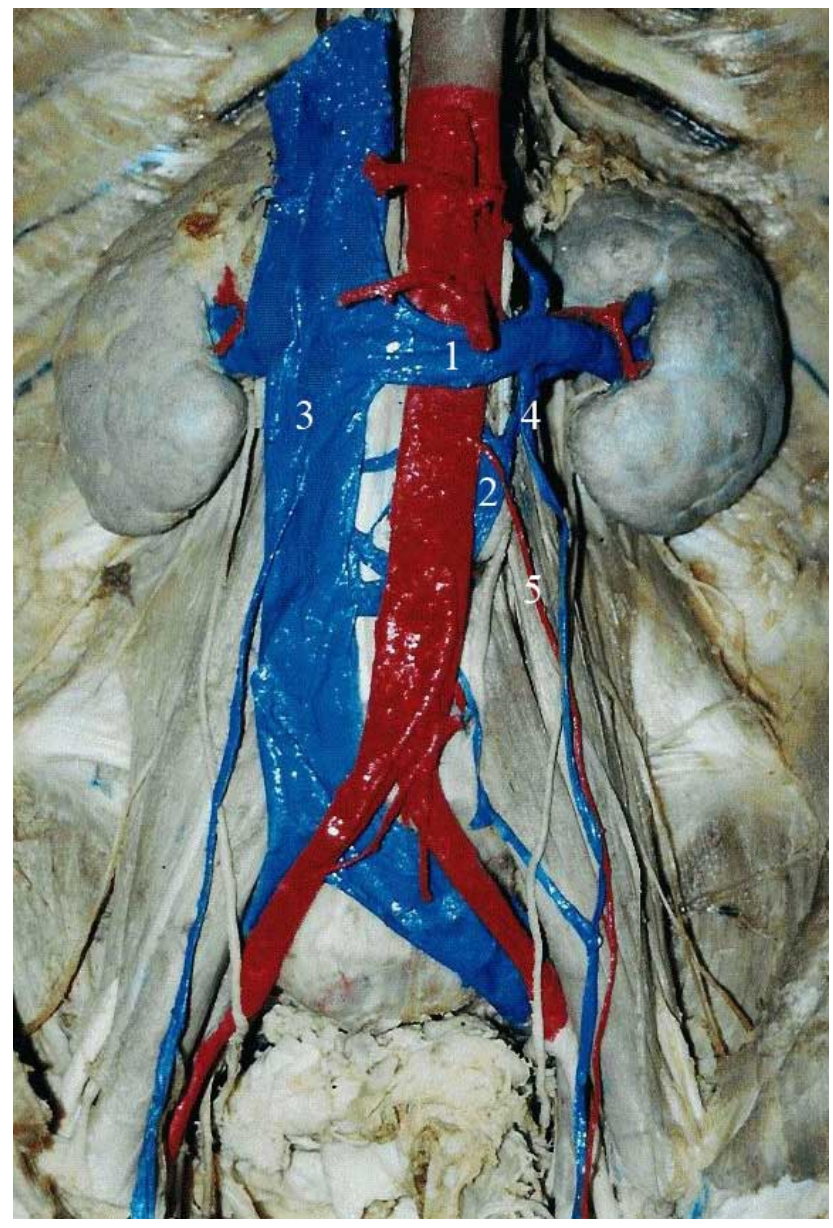

tenía un calibre de $14 \mathrm{~mm}$, posteriormente a la aorta. Antes de su desembocadura en la VCI, se dividió en dos ramas de calibre 6,0 y 9,0 mm, respectivamente (Fig. 2), que recorrían una distancia de 5,0 $\mathrm{mm}$.

Inmediatamente antes de la bifurcación de la vena renal izquierda retro-aórtica, ésta última recibió la $3^{\text {a }}$ vena lumbar izquierda y la vena lumbar ascendente izquierda. Las dos primeras venas lumbares izquierdas desembocaron por medio de un tronco común en la VCI a $15 \mathrm{~mm}$ superiormente al término de la vena renal izquierda retroaórtica.

La vena lumbar ascendente izquierda estaba localizada superficialmente al músculo psoas mayor, siendo formada por una afluente originada en la vena ilíaca común y por otra proveniente de la bifurcación de la vena testicular izquierda. La vena testicular izquierda poseía durante su trayecto, dos comunicaciones anómalas, una con la vena lumbar ascendente en formación y la otra con la arteria testicular homolateral.

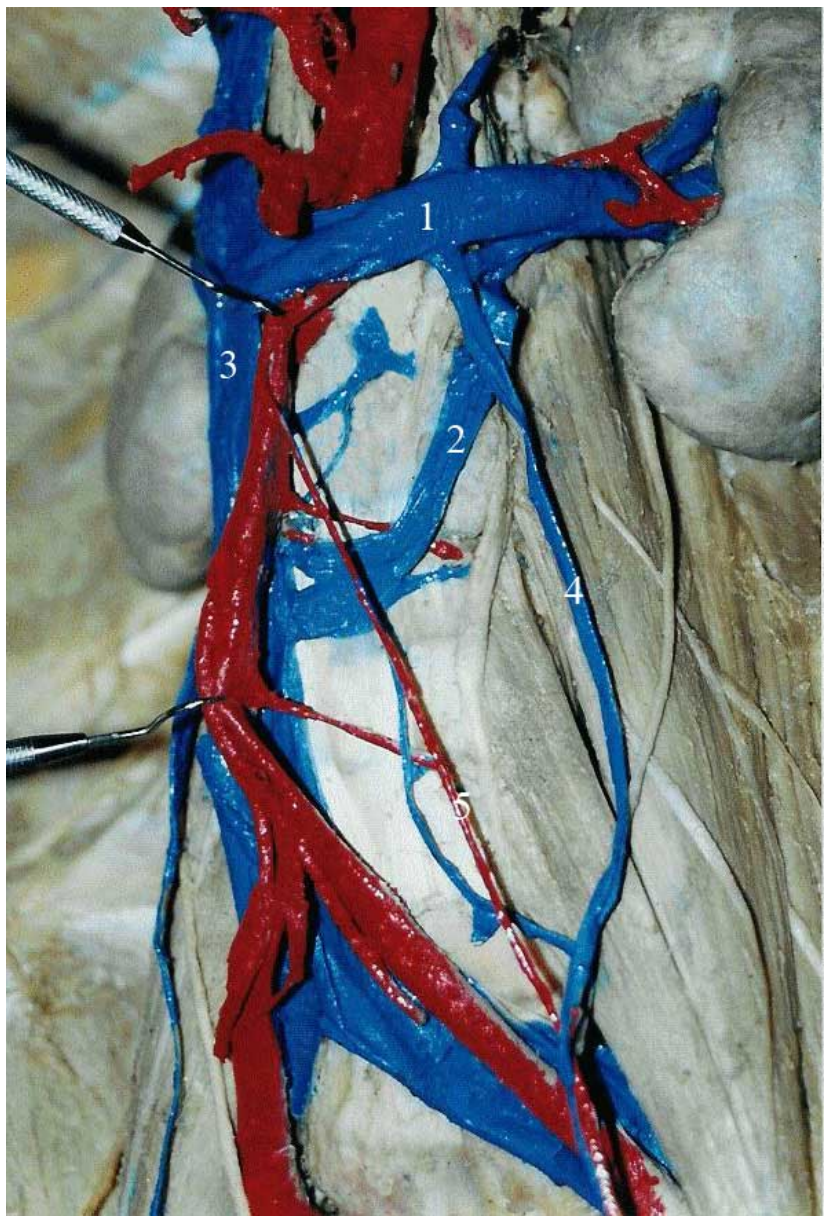

Figs. 1 y 2.- 1. Vena renal pre-aórtica; 2. Vena renal retro-aórtica; 3. Vena cava inferior; 4. Vena testicular izquierda; 5. Arteria testicular izquierda. 


\section{DISCUSIÓN}

La variaciones de la vena renal izquierda se clasifican en cuatro tipos de acuerdo a Karaman et al. (2007), donde en el tipo I el vaso ventral pre-aórtico de la vena renal izquierda está obliterado, persistiendo el vaso retro-aórtico, que se une a la vena cava inferior en posición ortóptica; el tipo II, resulta de la obliteración del vaso ventral pre-aórtico, permaneciendo el vaso dorsal que continua como vena renal izquierda retro-aórtica, que drena a nivel de las vértebras L4-L5; el tipo III corresponde a la vena renal izquierda circun-aórtica o collar venoso, y se debe a la persistencia de las anastomosis subsupracardinales e intersupracardinales y al vaso dorsal de la vena renal izquierda; en el tipo IV, el vaso ventral pre-aórtico de la vena renal izquierda está obliterado, permaneciendo el vaso dorsal que llegará a ser la vena renal izquierda, la que tendrá un trayecto oblicuo y caudal por detrás de la aorta y drenando en la vena ilíaca común. Estos autores señalaron que el tipo III, que correspondería a la variación presentada en este artículo, puede tener una incidencia de hasta $16 \%$.

La incidencia del collar renal es variable y ha sido descrita en $11 \%$ por Beckmann \& Abrams (1979) en una serie de 74 venogramas y en $0,3 \%$ por Satyapal et al. (1992) en 1008 disecciones, venogramas y estudios clínicos de pacientes sometidos a transplantes renales y cirugías de aneurisma de la porción abdominal de la aorta.

En relación a la embriogénesis, Babaian \& Johnson (1979) afirmaron que la formación de los varios segmentos de la vena cava inferior (VCI) involucra el surgimiento secuencial de tres canales venosos pareados: venas cardinales posteriores, supracardinales y subcardinales, así como el desarrollo de anastomosis entre ellos y la subsecuente aparición de cambios regresivos. Todo este proceso es normalmente completado alrededor de la $8^{\mathrm{a}}$ semana de gestación. Las primeras estructuras en aparecer son las venas cardinales posteriores, paralelas y longitudinales, localizadas en la parte dorsal del embrión y que durante el desarrollo, son sustituídas por cuatro canales longitudinales. Estos canales son las venas supracardinales pareadas, que son posteriores a la aorta y las venas subcardinales pareadas que son anteriores. Continua luego el desarrollo de ricas comunicaciones entre los canales supracardinales y subcardinales, las cuales forman un plexo venosos alrededor de la aorta.

Con frecuencia, la porción ventral del plexo circunaórtico (anastomosis intersubcardinal) persiste como la vena renal izquierda normal. Si la porción dorsal del plexo persiste, entonces habrá una vena renal izquierda, posterior a la aorta. Así, el componente dorsal del collar renal circun- aórtico se debe a la persistencia en el lado izquierdo de la anastomosis intersupracardinal embrionaria (Patten, 1962). Si las porciones ventral y dorsal persisten, habrá un collar venoso circun-aórtico en el adulto (Sobrinho, 1977).

El complejo desarrollo embrionario del sistema venoso cava, principalmente en su porción inferior (VCI y sus tributarias) da como resultado un grupo amplio de variaciones vasculares, las que deben ser recordadas por los cirujanos en procedimientos invasivos retroperitoneales como por ejemplo: en la intervenciones quirúrgicas por aneurisma aórtico abdominal, en nefrectomías, así como en la instalación de filtros en la VCI como prevención por la aparición de posibles tromboembolias derivadas de trombosis venosas profundas. La falta de reconocimiento de ese componente dorsal durante la cirugía retroperitoneal puede llevar a hemorragia, nefrectomía o muerte (Mitty, 1975; Babaian \& Johnson).

Tal es la importancia quirúrgica de esta variación, que Roditi et al. (1996) comentaron que procedimientos de imagenología tales como la resonancia mágnetica han sido usados cada vez más en evaluaciones pre-operatorias de la región y muchos centros utilizan varias técnicas para investigar previamente a los donadores vivos para transplante renal y evaluación de aneurismas de la aorta abdominal.

De acuerdo con Mitty, es de particular importancia que el imagenólogo reconozca el padrón de circulación asociado a esta variación durante inyecciones fluoroscópicas. Adicionalmente, el riñón izquierdo es usualmente preferido para transplante ya que la vena renal es más larga. Con el anillo circun-aórtico esa ventaja se pierde debido a su comunicación adicional a la VCI, llevando, quizás no a mayores complicaciones, sino a la necesidad de mayor cuidado en el procedimiento quirúrgico.

Babaian \& Johnson han citado que no reconocer la vena renal retro-aórtica puede resultar en una extensa disección quirúrgica en casos de linfadenectomía, realizada como procedimiento en el carcinoma testicular. Agregado a ello, cuando se realiza disección en el canal interaórtico y espacios paraaórticos, este vaso puede ser confundido como una vena lumbar o como una variante de la vena espermática y ser ligada o lesionada.

Un caso interesante de disposición retro-aórtica fue descrito por Inzunza et al. (2011), variación que se observa en aproximadamente $2 \%$ de la población, donde la vena renal izquierda se formó a partir de la unión de tres venas que 
emergían del hilio renal, donde desembocaron la vena gonadal izquierda y la suprarrenal, además de dos finos ramos lumbares y uno parietal. La vena renal retroáortica drenó junto con la vena ilíaca común izquierda en la vena cava inferior. En el año 2000, Brancatelli et al., presentaron un caso en que la vena renal izquierda, retro-aórtica, se unió a la vena ilíaca común izquierda, disposición semejante a la variación presentada por estos últimos autores.

En relación al anillo venoso circun-aórtico, Beckmann \& Abrams han señalado que éste puede ser un potencial camino para la trayectoria colateral de un émbolo pulmonar recurrente, proveniente de los miembros inferiores o de la pelvis, después de haber ligado a la vena cava inferior. Si durante una operación para interrupción de la circulación de la vena cava inferior, se encontrara el anillo venoso circun-aórtico, la ligadura debe ser realizada por debajo de la terminación de la vena renal retro-aórtica en la región lumbar inferior.

Las cirugías de aneurisma de la aorta abdominal poseen un problema particular, debido a que la vena renal izquierda es usada como punto de referencia debajo de la cual la aorta es clampeada. Como la porción posterior de ese anillo venoso pasa inferior y posteriormente, está expuesto a lesión durante el procedimiento mencionado (Mitty).

Observando la complejidad de las variaciones vasculares involucrando al "collar renal", concordamos con Mitty cuando señaló que la importancia del estudio y descripción del collar venoso circun-aórtico, reside en el hecho de ser una variación potencialmente peligrosa en cirugía.
En el presente estudio, las venas renales pre y retroaórtica se originaron separadamente del hilio renal, a diferencia de lo que fue relatado por Kramer (1977), Kramer \& Grine (1979) y quienes describieron diez casos donde emergía una sola vena, separándose luego en venas pre y retro-aórticas. Por su parte Nam et al. (2010) presentó una figura con los cuatro tipos, mostrando en el tipo III, un origen único para este vaso que luego se dividía en dos.

Por otra parte, la vena renal pre-aórtica era más desarrollada que la correspondiente retro-aórtica, siendo de $90 \mathrm{~mm}$ de longitud y $20 \mathrm{~mm}$ de calibre en su terminación. Beckmann \& Abrams señalaron que tal calibre varía de 11 a $22 \mathrm{~mm}$, con un promedio de 16,3 $\mathrm{mm}$. Estos mismos autores relataron que el diámetro de la vena renal retroaórtica varía de 4 a $13 \mathrm{~mm}$ con un promedio de 7,7 mm, valor coincidente con el informado en el presente trabajo, donde su calibre fue de $14 \mathrm{~mm}$ posteriormente a la aorta.

Con relación a la relación de estas venas con la vena gonadal izquierda, Beckmann \& Abrams describieron dos casos en que esta última vena drenó en la vena renal preaórtica y cuatro casos en que por medio de un tronco común lo hicieron próximo al hilio, donde las venas pre y retro-aórtica emergieron. En la variación presentada, la vena gonadal izquierda drenó en la vena pre-aórtica.

Sin duda, que el conocimiento de este tipo de variaciones venosas es de mucha importancia en los procedimientos quirúrgicos retroperitoneales, ya que la persistencia del collar venoso renal es de una incidencia importante.

SOUSA- RODRIGUES, C. F.; ALCÂNTARA, F. S.; ROCHA, A. C.; GUSMÃO, L. C.; MONTE-BISPO, R. F.; FERREIRA, A. K. \& OLAVE, E. Persistence of renal venous collar. Int. J. Morphol., 31(2):500-504, 2013.

SUMMARY: The development of the cava venous system is very complex, taking place to the raising of innumerous variations of the anatomical patterns already known, which could be more or less common and others, still, of rare incidence. One of these anomalies may occur in a low frequency on the left side, forming a vascular ring around the aorta constituted by a preaortic renal vein and other retro-aortic renal vein, entering in a lower level of the VCI, in a condition called as " Renal Collar" consisted of persistence of the Intersupracardial embrionary anastomoses. In this case report, the vascular disposition corresponds to a type of the classification as related in literature, with exception of the independent emergency of the preaortic and retro-aortic components of the circum-aortic collar from the renal hilum and the distal diameter of the retroaortic renal vein. The preaortic vein had $90 \mathrm{~mm}$ of length, diameter of $20 \mathrm{~mm}$ in its end and led into the VCI on the lower level of L1. The retroaortic vein measured $125 \mathrm{~mm}$ of length, crossing L1 and L2 to discharge in the VCI, to the upper level of L3, where it had diameter of $14 \mathrm{~mm}$. The importance of the study and description of the circumaortic renal collar is due to it representing a potentially hazardous anomaly of the left renal vein, occurring case reports where failure to recognize the dorsal component during retroperitoneal surgery may lead to abundant hemorrhage after inadvertent injury, unnecessary nephrectomy or, even death.

KEY WORDS: Kidney; Renal Veins; Anatomical variations 
SOUSA- RODRIGUES, C. F.; ALCÂNTARA, F. S.; ROCHA, A. C.; GUSMÃO, L. C.; MONTE-BISPO, R. F.; FERREIRA, A. K. \& OLAVE, E. Persistencia del collar venoso renal. Int. J. Morphol., 31(2):500-504, 2013.

\section{REFERENCIAS BIBLIOGRAFICAS}

Babaian, R.J. \& Johnson, D.E. Major Venous Anomalies Complicating Retroperitoneal Surgery. South. Medical J., 72(10):1254-8, 1979.

Beckmann, C.F. \& Abrams, H.L. Circumaortic Venous Ring: Incidence and Significance. Am. J. Roentgenology, 132: 561-5, 1979.

Brancatelli, G.; Galia, M.; Finazzo, M.; Sparacia, G.; Pardo, S. \& Lagalla, R. Retroaortic left renal vein joining the left common iliac vein. Eur. Radiol.,10(11):1274-5, 2000.

Gardner, E.; Gray, D. \& O’Rahilly; D. Anatomia: Estudo Regional do Corpo Humano. $4^{\circ}$ ed. São Paulo, Guanabara-Koogan, 1988.

Hollinshead W. H. Anatomía Humana. Rosario, La Medica, 1966.

Inzunza, H. O.; Inzunza, A.M. \& Salgado, A.G. Vena renal izquierda recurrente retroaórtica. Reporte de una rara variación. Int. J. Morphol., 29(2):339-43, 2011.

Karaman, B.; Koplay, M.; Ozturk, E.; Basekim, C.C.; Ogul, H.; Mutlu, H. et al. Retroaortic left renal vein: multidetector computed tomography findings and its clinical importance. Acta Radiol., 48:355-60, 2007.

Kramer, B. \& Grine, F.E. The Incidence of the Renal Venous Collar in South African Blacks. South African Medical J., 57:875-6, 1980.

Kramer, B. An Unusual Case of Persistent Renal Collar in a Human Adult Male. British J. Urol., 50:157-9, 1978.

Mitty, H.A. Circumaortic Renal Collar - A Potentially Hazardous Anomaly of the Left Renal Vein. Am. J. Roentgenology, 125(2):307-10, 1975.

Nam, J. K.; Park, S. W.; Lee, S. D. \& Chung, M. K. The clinical significance of a retroaortic left renal vein. Korean J. Urol., 51:276-80, 2010.

Patten, B.M. Embriología Humana. $4^{\circ}$ ed. Buenos Aires, El Ateneo, 1962.

Roditi, G.H.; Buff, B.L. \& Longmaid, H.E. MR Venography of Left Renal Vein Anomalies. Clin. Radiol., 51:861-4, 1996.
Satyapal, K.S.; Kalideen, J.M.; Haffejee, A.A.; Singh, B. \& Robbs, J.V. Left Renal vein variations. Surg. Radiol. Anat., 21(1):77-81, 1999.

Sobrinho, J.H.M. Embriologia e Taxonomia das Malformações Cardiovasculares. $1^{\circ}$ ed. São Paulo, Sarvier, 1977.

Williams, P.; Warwick, R.; Dyson, M. \& Bannister, L. Gray Anatomia. $37^{\circ}$ ed. São Paulo, Guanabara-Koogan, 1995.

Dirección para correspondencia:

Prof. Dr. Célio Fernando de Sousa Rodrigues

Disciplina de Anatomia Topográfica

Centro de Ciências Biológicas - UFAL

CEP: $57.010-060$

Praça Afrânio Jorge, s $/ n^{\circ}$ Prado

Maceió, AL

BRASIL

Email: celiofernando@yahoo.com.br

Recibido : 18-12-2012

Aceptado: 22-03-2013 